\title{
Comparison of Cube Rotations Around Axes Inclined Relative to the Environment or to the Cube
}

\author{
Margaret M. Shiffrar and Roger N. Shepard \\ Stanford University
}

\begin{abstract}
Observers judged whether 2 successive computer-displayed rotations of a cube were the same or different. With respect to the observers, each rotation was about a vertical axis ( $Y$ ), a horizontal (line-of-sight) axis (Z), an axis tilted just $10^{\circ}$ from vertical or horizontal, or a maximally oblique axis. Independently, with respect to the cube, each rotation was about a symmetry axis through opposite faces $(\mathrm{F})$ or through opposite corners $(\mathrm{C})$, an axis tilted $10^{\circ}$ from one of these symmetry axes, or an axis of extreme nonsymmetry. Speed and accuracy of comparison decreased as the axes of the successive rotations departed from the canonical axes of the environment $(Z$, or especially, $Y)$, or even more sharply, from the symmetry axes of the cube $(C$, or especially, $F)$. The internalized principles that guide the perceptual representation of rigid motions evidently are ones of kinematic geometry more than of physics.
\end{abstract}

Evidence from many sources has indicated that space is not psychologically isotropic. We and other animals are quickest and most accurate in detecting objects (e.g., Ogilvie \& Taylor, 1958 ) and in discriminating objects or their orientations (e.g., Alluisi, 1961; Lashley, 1938; Sutherland, 1957, 1969) when the objects have vertical or horizontal orientations. Moreover, vertical and horizontal directions furnish the principal cognitive framework with respect to which we recognize, classify, or compare objects (e.g., Attneave \& Curlee, 1977; Braine, 1978; Cooper \& Shepard, 1973; Corballis, Nagourney, Shetzer, \& Stefanatos, 1978; Hinton \& Parsons, 1981, 1988; Hock \& Trombly, 1978; Rock, 1973; Yin, 1969), detect their symmetries (e.g., Corballis \& Roldan, 1975; Leyton, 1986a, 1986b; Palmer \& Hemenway, 1978; Rock, 1973; Zimmer, 1984), and remember their orientations or relative locations in space (e.g., Franklin \& Tversky, 1990; Levine, Jankovic, \& Palij, 1982; Lynch, 1960; Mani \& Johnson-Laird, 1982).

In comparison with the vertical and horizontal directions, oblique or diagonal directions have typically been found to be less salient or effective (e.g., Appelle, 1972; Bryant, 1969; Olson, 1970; Olson \& Bialystok, 1983; Rudel \& Teuber, 1963). This phenomenon, known as the oblique effect, may result from a tendency to perceive diagonal lines as more

This research was supported by National Science Foundation Research Grant BNS 85-11685 to Roger N. Shepard. The experiment, initially proposed by Roger N. Shepard, was carried out by Margaret M. Shiffrar and was first reported as part of her First Year Project Report at Stanford University in June, 1986. Results from this experiment were also reported by her in April 1987 at the annual convention of the Western Psychological Association, Long Beach, California.

We are indebted to Brian Wandell for supplying both hardware and software, and to Andy Fitzhugh for further software development. Dennis Proffitt, Lynn Robertson, and an anonymous reviewer made helpful suggestions for the revision of an earlier version of this article.

Correspondence concerning this article should be addressed to Roger N. Shepard, Department of Psychology, Building 420, Stanford University, Stanford, California 94305-2130. vertical or horizontal than their actual orientations (Bouma \& Andriessen, 1968). The influential role of the vertical dimension may ultimately arise from the terrestrial gravitational field, which (as emphasized by Shepard, 1982, 1984) has remained an invariant of our physical environment throughout biological evolution.

In addition, an object may have its own intrinsic axes of symmetry, elongation, or customary orientation or motionaxes that the object carries with it as its orientation changes with respect to an external reference frame. Thus, such diverse objects as a person, a fish, a bee, or a car, alike, have an intrinsic front and back, left and right, and top and bottom (or, in the case of a person, head and feet) that are identifiable whether the object is standing in its canonical upright position, resting on its side, or turned completely upside down. Such intrinsic axes, too, play an important role in our perceptual organization, interpretation, and memory of objects and their mutual relations in space (e.g., Clark, 1973; Franklin \& Tversky, 1990; Leyton, 1986a, 1986b; Rock, 1973; Shepard, 1988; Shepard \& Hurwitz, 1984).

Most research has focused on the role of reference axes in the representation of static objects or static configurations of objects in space. Consideration has recently been shifting, however, to the possibility that the representation of the transformations of objects in space may be at least as fundamental as the representations of the objects themselves (e.g., Freyd, 1983, 1987; Leyton, 1986a, 1986b; Palmer, 1982, 1984; Shepard, 1981, 1984, 1988; Shepard \& Cooper, 1982). One motivation for such a shift is that, whereas the objects that have been significant for us and for our ancestors have differed along countless dimensions, the rigid transformations of those objects in three-dimensional space have had the same 6 degrees of freedom - three of translation and three of rotation-throughout biological evolution (Shepard, 1981, 1987).

If there are psychologically preferred axes with respect to an observer's environment and also with respect to an object itself, such preferred axes might determine which motions of the object are most readily imagined, are most often experienced in apparent motion, and are most quickly perceived 
and precisely remembered from an actual motion. For example, Post and Chaderjian (1987) found that the perceived path of a translating bar is influenced by the bar's orientation. In particular, the component of translation parallel to the long axis of the bar tended to be relatively overestimated. In further pursuing the phenomenon of mental rotation (Shepard \& Metzler, 1971), Metzler (1973) found early indications that three-dimensional objects were more efficiently compared when the objects differed by a rotation around one of their own natural axes and, also, when the axis of rotation was vertical in the environment (see Metzler \& Shepard, 1974; Shepard \& Cooper, 1982). Subsequent research has also found mental rotation to be more efficient when the axis of rotation is a natural axis of the object or of the environment (e.g., Friedman, Pilon, \& Gabrys, 1988; Just \& Carpenter, 1985; Parsons, 1987).

A possibly related finding is that bilateral symmetry, when detected, is highly salient and also that such symmetry is more readily detected when the axis of the symmetry is horizontal or, especially, vertical (e.g., Corballis \& Roldan, 1975; Kahn \& Foster, 1981, 1986; Palmer \& Hemenway, 1978; Rock, 1973). Finally, visual apparent motion, as well as imagined transformation, is evidently affected by the orientation of natural axes and by the symmetries of the object (e.g., Farrell \& Shepard, 1981; McBeath \& Shepard, 1989; Proffitt, Gilden, Kaiser, \& Whelan, 1988).

The experiment we report in this article focused on the perception and memory of real motion, as opposed to motion that is only imagined or apparent. Because the visual system has evolved for the representation of real motion, alternative real motions should offer ecologically appropriate probes of that system (e.g., see Cutting, 1986). Primarily, we sought evidence for principles governing our perceptual representation of rigid transformations of objects in space. Secondarily, we hoped that such principles might be understandable as accommodations to universal regularities of the world in which we have evolved (Shepard, 1984, 1987, 1988). In particular, we hoped to find some indication of whether our perceptual systems have more fully internalized the constraints of physics or the somewhat different constraints of kinematic geometry.

From the standpoint of physics, the only property of a body that is relevant for its motion is the inertially equivalent ellipsoid of its mass distribution. This property is specifiable in terms of the magnitudes and momentary directions of the body's three moments of inertia (which are aligned with the three major axes of its inertially equivalent ellipsoid). According to the laws of physics, the simplest motions of a body, those in which the direction of the axis of rotation remains constant, are those in which no external force acts on the body and in which any rotation is about a principal axis of the body's mass distribution or inertially equivalent ellipsoid (see Carlton \& Shepard, 1990a).

In contrast, geometry does not include such physical concepts as mass and inertia. From a geometrical standpoint, the only properties of an object that are relevant for its rotational motion are therefore the properties that can be specified purely in terms of the abstract, geometrical configuration of the object-particularly such properties as the object's (global or local) spatial symmetries (Carlton \& Shepard, 1990b; Leyton, 1986a, 1986b; Shepard \& Farrell, 1985).

Correspondingly, the object's simplest motions might then be those that leave the direction of one of the object's axes of symmetry invariant (i.e., rigid rotations about such an axis of symmetry).

Moreover, from a physical standpoint, the motion of a body that is not acted on by external forces is independent of the inertial frame of anyone observing that body's motion. But, from a geometrical standpoint, the characterization of an object and of its motion relative to the reference frame of an observer might be simpler when a symmetry axis of the object or the axis of any rotational component of the object's motion, or both, are aligned with a preferred direction in that reference frame. The specific constraints entailed by kinematic geometry and object symmetries are best formulated in terms of mathematical groups-in terms, respectively, of the Euclidean group of three-dimensional space (see Carlton \& Shepard, 1990a) and the symmetry group of the object (see Carlton \& Shepard, 1990b; Leyton, 1986a, 1986b).

For several reasons, we chose the cube as the object to undergo rigid motions. The cube is a particularly standard three-dimensional object. Two-dimensional projections of the cube are swiftly computed and displayed, in the form of straight-line segments on a computer-controlled vector scope. Rotations of the cube have already been analyzed from the standpoints of physics and kinematic geometry, that is, in terms of its inertially equivalent ellipsoid and its symmetry group, respectively (see Carlton \& Shepard, 1990a, 1990b).

According to the laws of physics, any unconstrained rigid body is dynamically equivalent to (i.e., it will freely move in space in exactly the same way as) an ellipsoid of uniform density_-called the equivalent ellipsoid of the given rigid body. The inertially equivalent ellipsoid of the cube is a sphere whose three principal axes and, hence, three moments of inertia are all equal. Consequently, the orientation of the principal axes of the cube is indeterminate. Uniform rotation about any axis through the center of the cube is therefore equally compatible with the laws of physics (Carlton \& Shepard, 1990a). Yet the cube, unlike a sphere or an arbitrary asymmetric shape, possesses intrinsic axes of symmetry around which rotational motions might be perceived as especially simple or natural by human observers. Specifically, the cube possesses three axes with fourfold symmetry (the axes through the centers of opposite faces), four axes with threefold symmetry (the axes through opposite corners) and, although these are not separately investigated in our experiment, six axes with twofold symmetry (the axes through the centers of opposite edges).

As the motions to be undergone by the cube, we chose pure rotations. Rotations are more complex, challenging, and general transformations than translations and, unlike pure translations, rotations offer the possibility of discriminating between the predictions of classical physics and kinematic geometry (see Carlton \& Shepard, 1990a). Second, pure rotations avoid the tendency of motions with a translational component to carry an object off the display screen.

Instead of asking observers to make subjective judgments of the psychological naturalness of different motions of the 
cube, we asked them to indicate whether the second of the two successively presented motions on each trial was objectively the same as or different from the first. We supposed that an observer's sensitivity to differences between two rotations would be greatest when those rotations were about psychologically natural axes. We further supposed that this sensitivity would be manifested as a greater speed and accuracy of the observer's classifications of such rotations as same or different.

\section{Method}

\section{Subjects}

Thirteen students from an introductory psychology course at Stanford University served as observers, in partial fulfillment of a course requirement. Nine of the observers were female, and 4 were male. All 13 were right-handed.

\section{Stimuli}

Each stimulus was a two-dimensional projection of a rotating cube, displayed on the screen of a computer-controlled vector scope (Hewlett-Packard Model 1345A) with a 2,048 × 2,048 pixel resolution and a $60 \mathrm{~Hz}$ refresh rate. The display of the cube was by parallel projection (i.e., without perspective convergence, as if the cube were viewed from an infinite distance), and observers viewed the cube binocularly. All 12 edges of the cube were represented as bright green lines with a uniform luminance of $170 \mathrm{~cd} / \mathrm{m}^{2}$, against the dark background of the screen. These edges were visible throughout the rotation except when momentarily occluded by other edges of the cube (e.g., see Cube F in Figure 2). The appearance was therefore of a luminous rotating "wire frame" cube (i.e., a cube with completely transparent faces). Throughout its rotation, the cube remained centered in the 9.5 by $12.5 \mathrm{~cm}$ display screen. The maximum lengths of the projected edges of the cube were $3 \mathrm{~cm}$ on the screen (whenever an edge was orthogonal to the line of sight). At the observer's distance from the screen of approximately $80 \mathrm{~cm}$, such edges subtended a maximum of about $2.2^{\circ}$ of visual angle. The whole cube subtended a visual diameter somewhere between $3^{\circ}$ and $4^{\circ}$ (depending on the momentary orientation of the cube). The cube was only displayed while rotating at the fixed rate of $187.5^{\circ} / \mathrm{s}$ about one of the specified axes in space. This angular velocity was chosen because it provided smooth motion of approximately one complete revolution of the cube during the display period. At the $60 \mathrm{~Hz}$ refresh rate, each momentary projection of the rotating cube was replaced every $16.7 \mathrm{~ms}$ by a new projection corresponding to a rotational increment of $3^{\circ}$ in the orientation of the cube. The high-resolution display yielded the appearance of a smooth, rigid rotation of the cube in three-dimensional space.

Rotations were always generated in the same direction (namely, clockwise, as viewed from above when the axis was vertical). The projection was susceptible to the reversals of depth interpretation familiar for such "Necker cubes," however, and to corresponding reversals in the apparent direction of rotation. During pilot studies, we found that the likelihood of the spontaneous reversals of the rotating cube increased with exposure duration. Specifically, spontaneous reversals were rare when a cube was seen to rotate for less than one revolution. We therefore tried to minimize spontaneous reversals by restricting the rotations to a maximum of $315^{\circ}$. We explained the possibility of reversals to observers and asked them not to consider such apparent reversals in the direction of rotation as constituting different rotations of the cube itself.
The differences in rotation of the cube itself (i.e., the true differences that the observers were asked to detect) were created by systematically varying the relation of a cube's axis of rotation to the observer's environmental frame or to the structure of the cube itself, or both. We included five similarly chosen ways in which the axis of rotation could be related to each of these two frameworks, namely, the framework of the environment and that of the cube. Specifically, we included (a) maximum alignment with each of two natural (environmental or object-centered) axes, (b) a small $\left(10^{\circ}\right)$ deviation from each of these natural axes, and (c) a maximum deviation from all such natural axes.

Specification of the axis of rotation relative to the environment. We assumed the natural axes of the observer's environment to be the vertical axis $(Y)$, the horizontal axis aligned with the observer's line of sight $(Z)$, and the horizontal axis orthogonal to that line of sight $(X)$-each separated from the other two by $90^{\circ}$. We included rotations around axes having five representative orientations relative to these three orthogonal axes-namely, rotations about (a) the vertical axis $(\mathrm{Y})$; (b), the line-of-sight axis $(\mathrm{Z})$; (c) a maximally oblique axis $(O)$ that was equally tipped (by $54.73^{\circ}$ ) from all three of the orthogonal axes, $X, Y$, and $Z$ (and, hence, that passed through the center of the equilateral triangle with vertices one unit out on Axes $X, Y$, and $Z$ ); (d) an axis (YO) tipped just $10^{\circ}$ (or about $18 \%$ of the way) from the vertical Axis $Y$ and towards the maximally oblique Axis $\mathrm{O}$; and (e) an axis ( $\mathrm{ZO}$ ) tipped just $10^{\circ}$ (or about $18 \%$ of the way) from the line-of-sight $A x i s ~ Z$ and toward the maximally oblique Axis $O$. (Thus, Axis YO lay in the plane defined by $Y$ and $O$, and Axis $Z O$ lay in the plane defined by $Z$ and $O$.) Figure la schematically illustrates the disposition of these five axes of rotation in space and, below, the intersections of these five axes with the equilateral triangle through $(1,1,1)$ on the orthogonal Axes X, Y, Z.

Specification of the axis of rotation relative to the object. After the cube was centered on a chosen environmentally specified axis of rotation, it could still be oriented in different ways with respect to that chosen axis. For example, the cube could be oriented so that the axis of rotation coincided with a fourfold symmetry axis through the centers of two opposite faces (F), a threefold symmetry axis through two diametrically opposite corners (C), or a twofold symmetry axis through the centers of two diagonally opposite edges (E). (The angles between three such neighboring axes would be approximately $54.7^{\circ}$ between the first and second, $45.0^{\circ}$ between the first and third, and $35.3^{\circ}$ between the second and third.) Alternatively, the cube could be oriented so that the axis of rotation is not aligned with any of these symmetry axes of the cube. Any such nonsymmetry axis would, however, fall in a triangular region between three such neighboring symmetry axes, F, C, and E (as indicated in Figure $1 \mathrm{~b}$ ).

In a manner parallel to that described for the environmentally specified axes of rotation, we selected five representative rotations of the cube relative to its own inherent structure-namely, rotations about (a) a symmetry axis through two opposite faces (F); (b) a symmetry axis through two diametrically opposite corners $(\mathrm{C})$; (c) an axis of maximal nonsymmetry $(\mathrm{N})$ that passed through the center of the triangular region defined by three neighboring symmetry axes (and that was separated from the $\mathrm{F}$ and $\mathrm{C}$ axes by about $34^{\circ}$ and $26^{\circ}$, respectively); (d) an axis (FN) tipped just $10^{\circ}$ (or about $29 \%$ of the way) from symmetry Axis $\mathrm{F}$ and towards nonsymmetry Axis $\mathrm{N}$; and (e) an axis (CN) tipped just $10^{\circ}$ (or about $38 \%$ of the way) from symmetry Axis $C$ and toward nonsymmetry Axis $N$. (Thus, $C N$ lay in the plane determined by $\mathrm{C}$ and $\mathrm{N}$, and Axis FN lay in the plane determined by $F$ and $N$.) Figure $1 b$ schematically illustrates the disposition of these five axes in space and, below, their intersections with the right triangular region on a face of the cube.

For cases in which the axis of rotation was vertical in the environment, Figure 2 illustrates three of the possible orientations of the cube relative to that axis-namely, those in which that rotation was 
a. Rotational Axes Y, Z, O, YO, \& ZC (in Relation to the Environment)

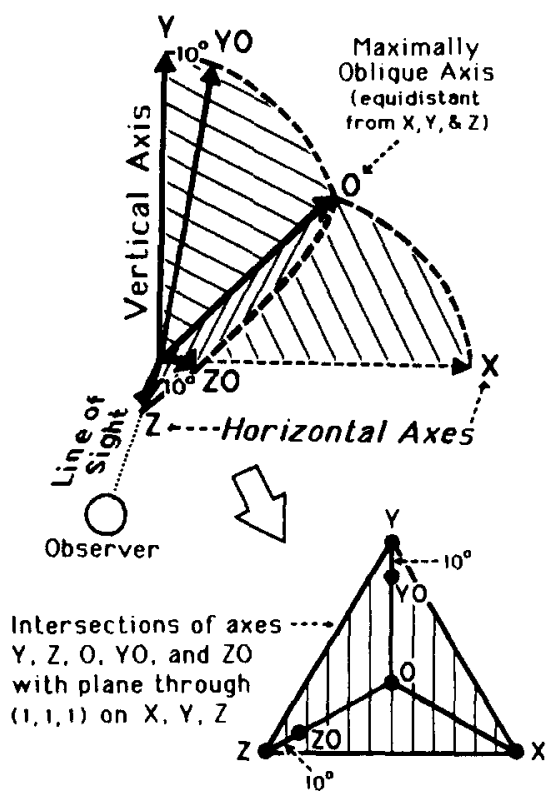

b. Rotational Axes F, C, N, FN, \& CN (in Relation to the Cube)

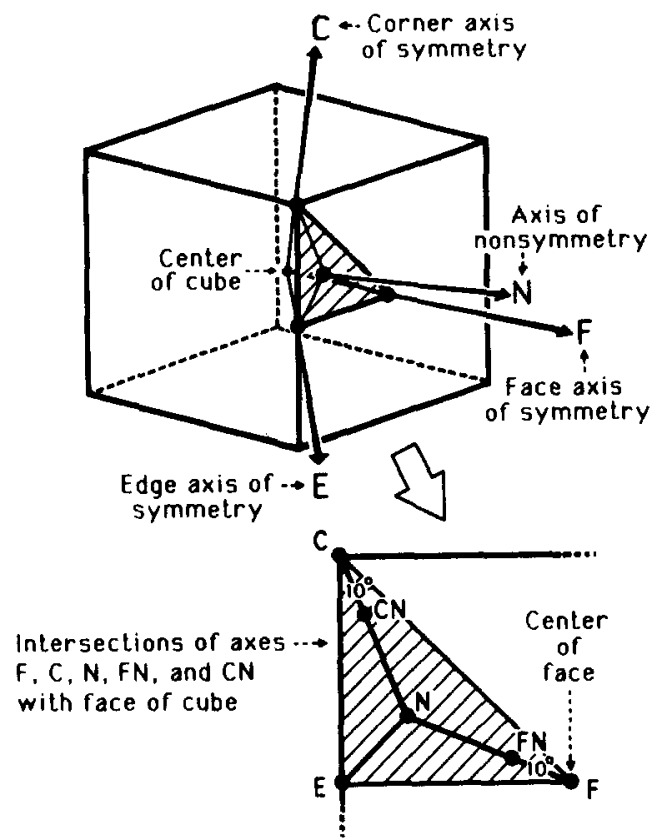

Figure 1. Schematic illustrations of the spatial dispositions (a) of the five rotational axes, Y, Z, O, YO, and $\mathrm{ZO}$, specified in relation to the observer's environment and (b) of the five rotational axes, F, C, N, $F N$, and $C N$, specified in relation to the cube itself.

about Axes $\mathrm{F}, \mathrm{C}$, and $\mathrm{N}$, relative to the cube. (The axis of rotation, indicated by the broken vertical line in this figure, was never itself displayed in the experiment.)

\section{Procedure}

Two rotations of the cube successively appeared on each trial. The experimenter instructed each observer to judge whether the two rotations were identical or whether they differed in any way (other than the merely apparent reversals attributable to spontaneous reversals in depth interpretation). The experimenter encouraged the observers to form a clear impression or "mental image" of the first rotation in each such pair in order to be able to compare it with the ensuing second rotation. Each observer first completed 25 practice trials. Following the instructions and this practice, all observers indicated that they understood and felt comfortable with the task. Each observer then completed four blocks of 37 experimental trials, for a total of 148 experimental trials.

A trial began when the observer pressed the key labeled next. After $1,500 \mathrm{~ms}$, the first of the two rotating cubes for that trial then appeared on the vector scope. The cube rotated around an axis randomly chosen from the five environmentally specified axes $(\mathrm{Y}, \mathrm{Z}, \mathrm{O}, \mathrm{YO}$, or $\mathrm{ZO}$ ). In addition, the axis of rotation had one of the five specified relations to the structure of the cube itself $(F, C, N, F N$, or $C N)$. This first rotation continued for $1,680 \mathrm{~ms}$, carrying the cube through a total angle of $315^{\circ}$. Then, following a 2-s blank screen, the second rotating cube appeared, also for $1,680 \mathrm{~ms}$. During this second rotation, the observer was to press the appropriate one of the other two keys, labeled same and different, to indicate whether this second rotation was the same as or different from the first rotation.

In one half of the trials, the two rotations were in fact identical (i.e., the cube started in the same orientation and rotated at the same rate about an axis having the same orientation with respect both to the environment and to the cube itself). In the other half of the trials, the two rotations differed in the relation of the axis of rotation to the environment, to the cube itself, or to both the environment and the cube. The observers were informed that the motions would be the same in one half and different in the other half of the trials. The observers were asked to register their decision about the pair of rotations in each trial "as quickly and accurately as possible during the display of the second cube." In analyzing the results, we excluded data from the initial practice trials and from those experimental trials in which an observer failed to make a same or different response during the $1,680 \mathrm{~ms}$ display of the second rotating cube. Such late responses occurred on less than $2 \%$ of all experimental trials.

As soon as the second cube disappeared from the vector scope, the (lower) display screen of the personal computer presented feedback about the accuracy and latency of the response made on that trial (e.g., Correct in 820 milliseconds). When observers were ready to begin the next trial, they again pressed the key marked next. Following completion of each 37-trial block (which typically required about 5 $\mathrm{min}$ ), the experimenter reentered the experimental room and initiated the next block of trials until all 148 experimental trials had been completed. The experimenter then asked the observer to describe any salient aspects of the stimuli or of the strategies the observer used to judge sameness or difference of the rotations. The experimenter also asked about the relative difficulties of comparing the different types of motions presented.

\section{Results}

On trials in which the two rotations were objectively identical, the overall mean percentage of correct same responses 
Alignments, with Vertical Axis of Rotation, of Axes of
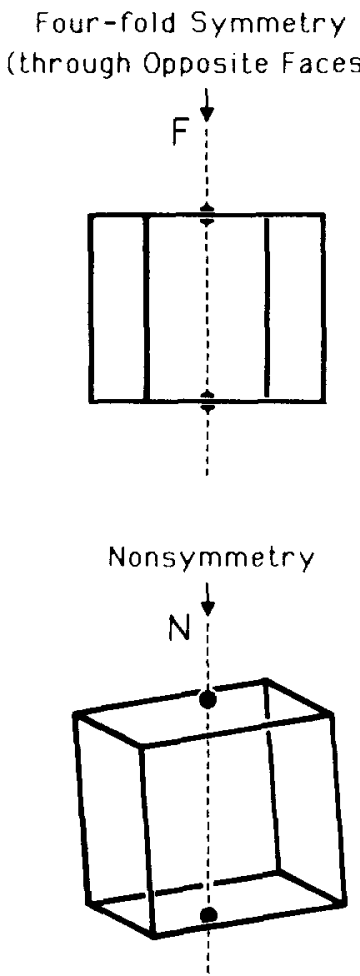

Three-fold Symmetry (through Opposite Corners)

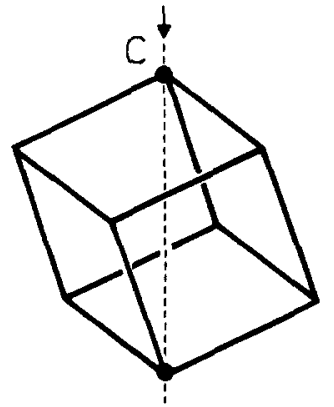

(Relation of nonsymmetry axis $\mathrm{N}$ to the three axes of symmetry, $F, C$, and $E$ )

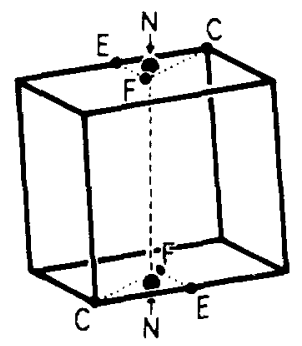

Figure 2. Orientations of the cube in which the axis of rotation coincides with the cube's face symmetry axis $\mathrm{F}$, with its corner symmetry axis $\mathrm{C}$, or with its nonsymmetry axis $\mathrm{N}$-illustrated for the environmentally vertical axis of rotation.

was $80.5 \%$, and the mean latency of these responses was 917 ms. As we had anticipated, the observers responded more quickly and accurately, however, when the rotational axis was aligned with a natural axis of the object or of the environment, or both. Because the data from all 13 observers manifested essentially the same pattern, the means we now report are averaged over the observers.

\section{Determinants of Latencies of Correct Same Responses}

Orientation of rotational axis relative to the environment. When the data were collapsed over the five different orientations of the cube relative to the axis of rotation, the latencies of correct same responses still differed significantly, depending on the orientation of that rotational axis relative to the environment, $F(4,48)=3.43, p<.02$. Among the mean latencies for individual environmentally specified axes, the longest, $978 \mathrm{~ms}$, was for the axis that was most oblique, O. As summarized in Table 1, linear contrasts indicated that latencies for rotations about this maximally oblique axis, $\mathrm{O}$, were significantly longer than latencies for rotations about three of the other four environmentally specified axes ( $Y, Z$, and $\mathrm{ZO}$ ). The shortest mean latency of a correct same response was for rotations about the environmentally vertical axis, $Y$. The three mean latencies for rotations about Axes $Z$,

YO, and $\mathrm{ZO}$ were intermediate in value and did not significantly differ among themselves.

Orientation of rotational axis relative to the object. When the data were collapsed, instead, over the five different orientations of the rotational axis relative to the environment, the latencies of correct same responses still differed significantly, depending on the orientation of the cube relative to that rotational axis, $F(4,48)=13.3, p<.001$. The longest mean latency, $1,019 \mathrm{~ms}$, was obtained for rotation about the least symmetric axis of the cube itself $(N)$. As summarized in Table 2, linear contrasts indicated that latencies for rotations about this most nonsymmetric axis, $N$, were significantly longer than latencies for rotations about each of the other cube-centered axes (F, C, FN, and $\mathrm{CN}$ ). Rotations yielding the shortest mean latency for the response same were those about the fourfold symmetry axis, $F$, through opposite faces of the cube $(816 \mathrm{~ms})$. Indeed, as shown at the top of Table 2, the mean latency for rotations about this axis, F, was significantly shorter than the latencies for rotations about each of the other four tested axes (C, N, FN, and CN). In particular, the latency for rotations about Axis $F$ was significantly shorter than for rotations about Axis $\mathrm{FN}(p<.001)$, which was tipped away from it by only $10^{\circ}$. The mean latency for rotations about the threefold symmetry axis, $\mathrm{C}$, through opposite corners did not differ significantly from that for rotations about Axis $\mathrm{CN}$, although these two axes also differed by $10^{\circ}$ (and although latencies for rotations about the two intermediate axes, FN and $\mathrm{CN}$, did not differ significantly from each other). Observers appear to be more sensitive to a small deviation from an axis of fourfold symmetry than to the same small deviation from an axis of only threefold symmetry.

Relative importances of and interaction between these two factors. Comparison of Tables 1 and 2 indicates that latencies of correct same responses increased more sharply as the rotational axis departed from the most symmetric axis of the cube than as the rotational axis departed from the canonical axes of the environment. Thus, for the environmental axes, the latency difference between rotations around the most preferred, vertical axis (Y) and the least preferred, oblique axis (O) was $96.5 \mathrm{~ms}$, with a $t$ value of 3.37 (and $p<.01$ ); whereas for the cube-centered axes, the latency difference between rotations around the most preferred, fourfold symmetry axis (F) and least preferred, most nonsymmetric axis (N) was $203.5 \mathrm{~ms}$, an increase of over $200 \%$, with a correspondingly increased $t$ value of 7.02 (and $p<.001$ ). Moreover, this is despite the fact that the angular difference between the two environmentally specified axes in the first pair (about $55^{\circ}$ ) was considerably larger than the angular difference between the two cube-centered rotational axes in the second pair (about $34^{\circ}$ ). Further, the increase in latency associated with a $10^{\circ}$ deviation of the rotational axis from the environmentally vertical axis (Y) was only $39.6 \mathrm{~ms}$ and nonsignificant, $t(12)=1.38, p>.20$, whereas the increase in latency accompanying the same $10^{\circ}$ deviation of the rotational axis from the fourfold symmetry axis through opposite faces of the cube $(F)$ was $131.8 \mathrm{~ms}$, an increase of more than $300 \%$, and highly significant, $t(12)=4.57, p<.001$.

Figure 3 summarizes the joint effect, on latency of correct same response, of deviation of the rotational axis from the natural axes of both the environment and the cube. For each 
Table 1

Dependencies of Latencies of the Correct Same Response and of Accuracies on the Orientation of the Rotational Axis With Respect to the Environment (When the Two Successive Rotations Were Around the Same Axis)

\begin{tabular}{|c|c|c|c|c|c|c|}
\hline \multirow[b]{2}{*}{ Axis } & \multirow[b]{2}{*}{$\begin{array}{l}\text { Mean latency } \\
\text { (in ms) }\end{array}$} & \multirow[b]{2}{*}{ \% Same } & \multirow[b]{2}{*}{$d^{\prime}$} & \multirow[b]{2}{*}{$\begin{array}{l}\text { Comparison } \\
\text { axis }\end{array}$} & \multicolumn{2}{|c|}{ Linear contrast } \\
\hline & & & & & $\begin{array}{l}t \text { value } \\
(12 d f)\end{array}$ & $\begin{array}{c}\text { Significance } \\
\text { (2-tailed prob.) }\end{array}$ \\
\hline $\begin{array}{c}\mathrm{Y} \\
\text { (vertical axis) }\end{array}$ & 881.5 & 83.0 & 1.41 & $\begin{array}{l}\mathrm{Z} \\
\mathrm{O} \\
\mathrm{YO} \\
\mathrm{ZO}\end{array}$ & $\begin{array}{r}-0.99 \\
3.37 \\
1.38 \\
0.34\end{array}$ & $\begin{array}{c}n s \\
p<.01 \\
n s \\
n s\end{array}$ \\
\hline$\frac{\mathrm{Z}}{\text { (line-of-sight axis) }}$ & 909.8 & 85.1 & 1.31 & $\begin{array}{l}\text { Y } \\
\text { O } \\
\text { YO } \\
\text { ZO }\end{array}$ & $\begin{array}{r}-0.99 \\
2.42 \\
0.40 \\
-0.61\end{array}$ & $\begin{array}{l}n s \\
p<.05 \\
n s \\
n s\end{array}$ \\
\hline $\begin{array}{l}\mathrm{O} \\
\text { (oblique axis far from } \\
\text { axes } \mathrm{X}, \mathrm{Y}, \mathrm{Z} \text { ) }\end{array}$ & 978.0 & 74.2 & 0.92 & $\begin{array}{l}\mathrm{Y} \\
\mathrm{Z} \\
\mathrm{YO} \\
\mathrm{ZO}\end{array}$ & $\begin{array}{r}3.37 \\
2.42 \\
-2.00 \\
-2.92\end{array}$ & $\begin{array}{c}p<.01 \\
p<.05 \\
n s \\
p<.02\end{array}$ \\
\hline $\begin{array}{l}\quad \mathrm{YO} \\
\text { (axis tipped } 10^{\circ} \text { from } \\
\text { vertical axis, } \mathrm{Y} \text { ) }\end{array}$ & 921.1 & 79.6 & 1.21 & $\begin{array}{c}\mathrm{Y} \\
\mathrm{Z} \\
\mathrm{O} \\
\mathrm{ZO}\end{array}$ & $\begin{array}{r}1.38 \\
0.40 \\
-2.00 \\
0.99\end{array}$ & $\begin{array}{l}n s \\
n s \\
n s \\
n s\end{array}$ \\
\hline $\begin{array}{l}\mathrm{ZO} \\
\text { (axis tipped } 10^{\circ} \text { from } \\
\text { line-of-sight, } \mathrm{Z} \text { ) }\end{array}$ & 891.8 & 81.1 & 1.23 & $\begin{array}{c}\mathrm{Y} \\
\mathrm{Z} \\
\mathrm{O} \\
\mathrm{YO}\end{array}$ & $\begin{array}{r}0.34 \\
-0.61 \\
-2.92 \\
-0.99\end{array}$ & $\begin{array}{c}n s \\
n s \\
p<.02 \\
n s\end{array}$ \\
\hline
\end{tabular}

level of each kind of deviation, latency increased monotonically with the other type of deviation. Again, however, deviation from the most symmetric axes of the cube had the stronger effect. Across all deviations from the canonical axes of the environment the three mean latencies for a $0^{\circ}$ departure from symmetry $(810,854$, and $909 \mathrm{~ms})$ were all shorter than each of the three mean latencies for a $10^{\circ}$ departure from symmetry $(930,943$, and $948 \mathrm{~ms})$; and these, in turn, were all shorter than each of the three mean latencies for a maximum departure from symmetry $(995,1,012$, and $1,035 \mathrm{~ms})$.

In addition to the greater effect of deviation from the most symmetric axes of the cube, there was a statistically significant

Table 2

Dependencies of Latencies of the Correct Same Response and of Accuracies on the Orientation of the Rotational Axis With Respect to the Cube (When the Two Successive Rotations Were Around the Same Axis)

\begin{tabular}{|c|c|c|c|c|c|c|}
\hline \multirow[b]{2}{*}{$\begin{array}{l}\text { Axis } \\
\text { (relative to the cube) }\end{array}$} & \multirow[b]{2}{*}{$\begin{array}{l}\text { Mean latency } \\
\text { (in ms) }\end{array}$} & \multirow[b]{2}{*}{ \% Same } & \multirow[b]{2}{*}{$d^{\prime}$} & \multirow[b]{2}{*}{$\begin{array}{c}\text { Comparison } \\
\text { axis }\end{array}$} & \multicolumn{2}{|c|}{ Linear contrast } \\
\hline & & & & & $\begin{array}{l}t \text { value } \\
(12 d f)\end{array}$ & $\begin{array}{c}\text { Significance } \\
\text { (2-tailed prob.) }\end{array}$ \\
\hline $\begin{array}{l}\text { F } \\
\text { (symmetry axis } \\
\text { through opposite faces) }\end{array}$ & 815.7 & 82.0 & 1.83 & $\begin{array}{c}\mathrm{C} \\
\mathrm{N} \\
\mathrm{FN} \\
\mathrm{CN}\end{array}$ & $\begin{array}{l}2.82 \\
7.02 \\
4.57 \\
3.67\end{array}$ & $\begin{array}{l}p<.02 \\
p<.001 \\
p<.001 \\
p<.01\end{array}$ \\
\hline \begin{tabular}{l}
\multicolumn{1}{c}{ C } \\
(symmetry axis \\
through opposite cor- \\
ners)
\end{tabular} & 893.3 & 92.8 & 1.65 & $\begin{array}{l}\mathrm{F} \\
\mathrm{N} \\
\mathrm{FN} \\
\mathrm{CN}\end{array}$ & $\begin{array}{l}2.82 \\
4.54 \\
1.97 \\
0.94\end{array}$ & $\begin{array}{c}p<.02 \\
p<.001 \\
n s \\
n s\end{array}$ \\
\hline $\begin{array}{l}\mathrm{N} \\
\text { (nonsymmetry axis far } \\
\text { from symmetry axes) }\end{array}$ & $1,019.2$ & 69.3 & 1.02 & $\begin{array}{l}\text { F } \\
\text { C } \\
\text { FN } \\
\text { CN }\end{array}$ & $\begin{array}{r}7.02 \\
4.54 \\
-2.46 \\
-3.44\end{array}$ & $\begin{array}{l}p<.001 \\
p<.001 \\
p<.05 \\
p<.01\end{array}$ \\
\hline $\begin{array}{l}\text { FN } \\
\text { (axis tipped } 10^{\circ} \text { from } \\
\text { face axis, F) }\end{array}$ & 947.5 & 76.8 & 1.16 & $\begin{array}{c}\mathrm{F} \\
\mathbf{C} \\
\mathrm{N} \\
\mathrm{CN}\end{array}$ & $\begin{array}{r}4.57 \\
1.97 \\
-2.46 \\
-1.03\end{array}$ & $\begin{array}{c}p<.001 \\
n s \\
p<.05 \\
n s\end{array}$ \\
\hline $\begin{array}{l}\mathrm{CN} \\
\text { (axis tipped } 10^{\circ} \text { from } \\
\text { corner axis, C) }\end{array}$ & 918.5 & 80.0 & 1.00 & $\begin{array}{l}\mathrm{F} \\
\mathrm{C} \\
\mathrm{N} \\
\mathrm{FN}\end{array}$ & $\begin{array}{r}3.67 \\
0.94 \\
-3.44 \\
-1.03 \\
\end{array}$ & $\begin{array}{c}p<.01 \\
n s \\
p<.01 \\
n s\end{array}$ \\
\hline
\end{tabular}




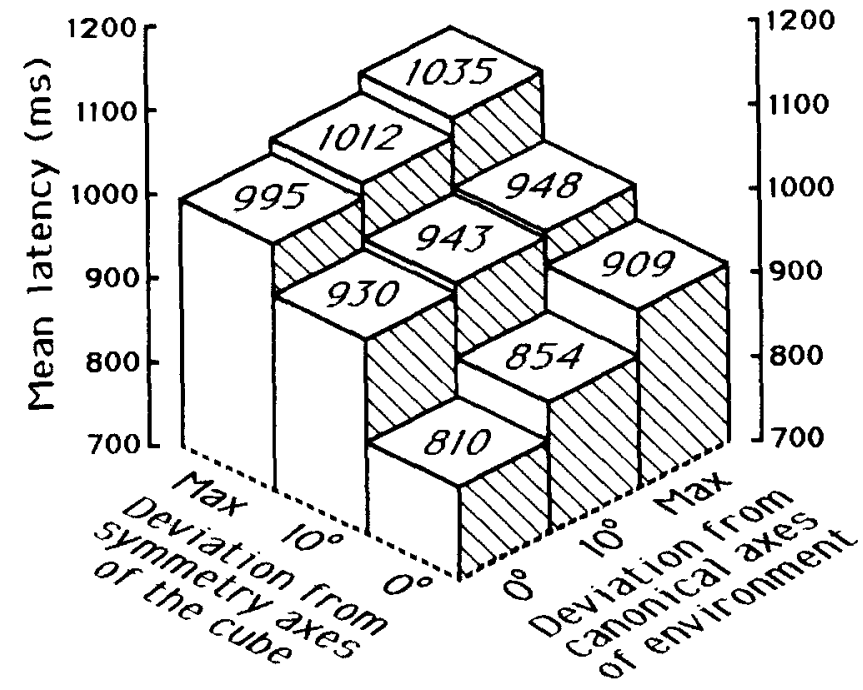

Figure 3. Latencies of correct same responses as a joint function of deviation of the rotational axis from the canonical axes ( $\mathrm{Y}$ and $\mathrm{Z}$ ) of the environment and from the symmetry axes ( $F$ and $C$ ) of the cube.

interaction between the effects of the two types of deviations, $F(8,48)=3.20, p<.025$. Figure 3 indicates that when the rotational axis was exactly aligned with a threefold or fourfold symmetry axis of the cube, speed of responding became not only faster but also more sharply dependent on deviation of the rotational axis from the canonical axes of the environment.

\section{Determinants of Accuracies for Same Responses}

Orientation of rotational axis relative to the environment. For each of the environmentally specified axes, the second column of Table 1 includes, next to the corresponding mean latency of same response, accuracy data in two forms: the percentage of correct same responses and a computed $d^{\prime}$ measure of discrimination. Both accuracy measures corroborate the latency results in indicating that rotations were more effectively compared when the rotations were around axes that were more closely aligned with environmentally natural axes. Among the five environmentally specified rotational axes, the canonical environmental axes ( $\mathrm{Y}$ and $\mathrm{Z}$ ) yielded the highest percentages of correct same responses $(83 \%$ and $85 \%$, respectively) and the highest values of $d^{\prime}(1.41$ and 1.31 , respectively). The most oblique axis, $\mathrm{O}$, yielded the lowest percentage of correct same responses $(74 \%)$ and the lowest value of $d^{\prime}(0.92)$. Measures of accuracy of both kinds were intermediate for the two intermediate axes, YO and $\mathrm{ZO}$.

Orientation of rotational axis relative to the object. The accuracy data for the five axes specified in relation to the cube are similarly presented in Table 2 . Among these five axes, the two most symmetric axes ( $\mathrm{F}$ and $\mathrm{C})$ yielded the highest percentage of correct same responses $(82 \%$ and $93 \%$, respectively) and the highest values of $d^{\prime}(1.83$ and 1.65 , respectively). The most nonsymmetric axis, $\mathrm{N}$, yielded the lowest percentage of correct same responses $(69 \%)$, and the inter- mediate axes ( $\mathrm{CN}$ and $\mathrm{FN}$ ) again yielded intermediate percentages correct ( $80 \%$ and $77 \%$, respectively). The $d^{\prime}$ measure was anomalously low for the $\mathrm{CN}$ axis, however, and indeed, lower than for the axis of maximal nonsymmetry, N. This anomaly appears to be the consequence of a relatively high proportion of false alarms for this particular axis type.

As in the case of the latency data, the difference between the accuracy data for the canonical axes $(Y, Z, F$, and $C)$ and the axes that deviated farthest from those canonical axes $(O$ and $\mathrm{N}$ ) was greater when the axes were specified in relation to the cube (a difference of 18 in percentage of correct same responses and of 0.65 in $d^{\prime}$ ) than when the axes were specified in relation to the environment (a difference of 10 in percentage of correct same responses and of 0.46 in $d^{\prime}$ ). Although the greater percentage of correct same responses for rotations about the cube's threefold symmetry axis $(C)$ than for rotations about its fourfold symmetry axis $(\mathrm{F})$ appears as a departure from parallelism between the latency and accuracy data, the $d^{\prime}$ measure of accuracy is in agreement with the latency data in indicating better performance when rotations were about the $\mathrm{F}$ axis $\left(d^{\prime}=1.83\right)$ than about the $\mathrm{C}$ axis $\left(d^{\prime}=1.65\right)$.

\section{Determinants of Latencies for Correct Different Responses}

In agreement with the pervasive tendency of discriminative reaction time to decrease with the degree of difference between the stimuli to be discriminated, the latencies of correct different responses in our task decreased with the degree of angular difference between the two successively presented rotations. Thus, with respect to the environmental axes, if the two rotations of a trial differed by $44.7^{\circ}$ (i.e., the pair O-YO in either order or the pair O-ZO in either order), then the average latency of correct different responses was $187 \mathrm{~ms}$ longer than if the rotational axes differed by $54.7^{\circ}$ (i.e., the pairs $\mathrm{O}-\mathrm{Y}$ or $\mathrm{O}-\mathrm{Z}$ ). This $187 \mathrm{~ms}$ difference (979 ms vs. $792 \mathrm{~ms}$ ) was significant, $t(12)=4.68, p<.001$. The same pattern of results holds for different trials containing rotations about the vertical and horizontal axes of the environment. If two rotations differed by $10^{\circ}$ (i.e., the pairs $\mathrm{Z}-\mathrm{ZO}$ or $\mathrm{Y}-\mathrm{YO}$ ), the average latency of correct different responses was $904 \mathrm{~ms}$ but only 792 $\mathrm{ms}$ if the rotations differed by $54.7 \mathrm{~ms}$ (i.e., the pairs $Z-O$ or $\mathrm{Y}-\mathrm{O})$. This $112 \mathrm{~ms}$ difference fell short of statistical significance, however, $t(12)=2.14, p<.065$.

The effect of angular difference on response latency was also found when the data were analyzed in terms of the symmetry axes of the cube itself. Thus, if the orientations of the two rotations of a trial differed by $10^{\circ}$ (i.e., F-FN or C$\mathrm{CN}$ ), then the average correct response latency was longer than if the orientations differed by $35^{\circ}$ (i.e., $\mathrm{F}-\mathrm{N}$ or $\mathrm{C}-\mathrm{N}$ ), although this $33 \mathrm{~ms}$ difference was not significant, $t(12)=$ $1.29, p<0.25$. Moreover, if two orientation axes differed by $25^{\circ}$ (i.e., $\mathrm{N}-\mathrm{CN}$ or $\mathrm{N}-\mathrm{FN}$ ), then the average response latency was $1,067 \mathrm{~ms}$ but only $866 \mathrm{~ms}$ if the orientations differed by $35^{\circ}$ (i.e., $\mathrm{N}-\mathrm{F}$ or $\mathrm{N}-\mathrm{C}$ ). This $201 \mathrm{~ms}$ difference was significant, $t(12)=6.13, p<.001$.

Overall, the longest response latencies for correct different responses were for trials in which both the first and second 
cube had oblique or nonsymmetry axes of rotation. The mean latency for two rotations about environmentally oblique axes (i.e., O, YO, or ZO) was $979 \mathrm{~ms}$, which was significantly longer than the mean latency of $848 \mathrm{~ms}$ when only one of the two rotations was about an oblique axis, $t(12)=3.84, p<.01$. Similarly, the mean latency for two rotations about axes that were not symmetry axes of the cube (i.e., $\mathrm{CN}, \mathrm{FN}$, or $\mathrm{N}$ ) was $1,067 \mathrm{~ms}$, which was significantly longer than the mean latency of $880 \mathrm{~ms}$ when only one rotation was about such an axis, $t(12)=6.4, p<.001$.

When the two rotations presented on a trial were objectively different, response latency depended on the type of axis presented first. If the first rotation was about a highly symmetric axis of the cube or a natural axis of the environment, then observers were able to make discriminations more rapidly. For example, if the first rotation was about a symmetry axis of the cube ( $\mathrm{F}$ or $\mathrm{C}$ ), then the mean latency of correctly responding different was only $829 \mathrm{~ms}$. On the other hand, if the first rotation was about one of the nonsymmetry axes $(\mathrm{N}$, $\mathrm{FN}$, or $\mathrm{CN}$ ), then the average response latency of $922 \mathrm{~ms}$ was significantly longer, $t(12)=3.59, p<.01$. Further, when the first rotation was about a fourfold symmetry axis, then average correct different response latency was significantly shorter than when the first rotation was about a threefold symmetry axis, $t(12)=3.94, p<.002$. Once again, this same pattern also held with respect to the environmental axes of rotation. For example, when the first rotation was about the environmental axis, $Y$, the mean response latency for correct different responses was significantly shorter than when the first rotation was about one of the three oblique environmental axes, $t(12)=2.89, p<.02$

\section{Observers' Self-Reports About the Experimental Task}

After completing the experimental trials, observers reported that they had been largely successful in remembering the first rotation of the cube on each trial and in comparing the second rotation with their "memory image" of that first rotation. A few observers reported occasional spontaneous reversals of depth and, hence, of apparent direction of rotation of the cube. They indicated that these reversals were readily recognizable as subjective, however, and did not significantly interfere with their determinations of whether the two rotations were objectively about the same or about different axes. Moreover, most observers reported experiencing no such reversals during the experimental trials. Observers also indicated that the first rotation could be more precisely remembered and the two rotations could be more readily compared when the first rotation was about the (environmentally) vertical or horizontal axis and, also, when that rotation was about a natural axis of the cube itself. In particular, some observers described rotations that were not about a symmetry axis of the cube as exhibiting a "wobble," even in the cases in which the rotational axis ( $\mathrm{FN}$ or $\mathrm{CN}$ ) departed by only $10^{\circ}$ from one of the symmetry axes ( $\mathrm{F}$ or $\mathrm{C}$ ). They indicated that the axis of such rotations did not have as clearly fixed or determinate an orientation in space.

\section{Discussion}

The results of this study appear to be clear and readily interpretable. In deciding whether two successively displayed rotations of a cube were the same or different, observers were consistently faster and more accurate when the axes of the rotations were aligned with the natural axes of the environment or the cube, or both. Latencies were shortest of all when the rotational axis was aligned with the environmental vertical and, particularly, when the rotational axis was aligned with a fourfold symmetry axis through opposite faces of the cube itself.

That the observers perceived rotations about obliquely oriented axes with less speed and accuracy than rotations about horizontal or vertical axes suggests that the oblique effect, which is now well established for the perception and comparison of the orientations of stationary objects, plays an important role in the perception of motions of objects as well. Such an extension of the oblique effect was previously suggested by studies of mental rotation, in which performance was somewhat reduced when the objects to be compared differed by a rotation about an environmentally arbitrary rather than about a vertical axis (e.g., Friedman et al., 1988; Metzler \& Shepard, 1974; Parsons, 1987). The detection of bilateral symmetry, too, has generally been reported to be best when the axis of the symmetry is vertical (e.g., Kahn \& Foster, 1981, 1986; Palmer \& Hemenway, 1978; Rock, 1973).

The superiority of nonoblique axes must ultimately derive from the anisotropy that the earth's gravitational field has uniformly conferred on the terrestrial environment. This invariant field may also have given rise to a number of psychologically relevant concomitants (e.g., Clark, 1973; Shepard \& Hurwitz, 1984). Thus, relative to the gravitationally determined upward direction, we are constrained to move about on the surface of a relatively flat supporting surface below; natural illumination of our surroundings most often comes from above; our usual locomotions cause what is in front or behind and to right or left to change from moment to moment, while what is above our heads or below our feet tends to remain relatively constant. Finally, through natural selection, the existence and invariance of the gravitationally determined unique upward direction and its concomitants may have come to be internally represented in many evolutionary lines, including our own.

Our concern here has been with the alignment of the axis of rotation with the natural axes of the observer and the environment, when these two sets of natural axes retain their standard alignment with each other. We have not attempted to separate the effects of departures from each of these two types of alignment, as when an observer is systematically tilted or rotated with respect to the environment. Our choice of specifying the axis of rotation in relation to the environment (or to the "observer's environment") rather than in relation to the observer was largely based on previous evidence suggesting that the fixed environmental frame would probably take cognitive priority over the observer's momentary frame if these were put into conflict with each other (cf. Attneave \& Olson, 1967; Corballis et al., 1978; Hinton \& Parsons, 1988; Rock, 1973; Rock \& Heimer, 1957; Rock \& Leaman, 1963). 
Nevertheless, further experiments may discover systematic differences between retinal and environmental coordinates with respect to the perception and representation of rotation.

The variable having the largest effect on performance in our experiment was the degree of departure of the axis of rotation from the symmetry axes of the cube itself-especially (according to the latency data), departure from an axis through opposite faces, F, an axis having greatest (i.e., fourfold) symmetry. This finding is consistent with evidence for the importance of symmetries and natural axes in many perceptual tasks and, in particular, in tasks of mental rotation (e.g., Friedman et al., 1988; Just \& Carpenter, 1985; Metzler \& Shepard, 1974), apparent motion (e.g., Farrell \& Shepard, 1981; McBeath \& Shepard, 1989; Proffitt et al., 1988), and translation (Post \& Chaderjian, 1987). There are two possible bases for the role of symmetry.

First, alignment of the axis of rotation with an axis of symmetry generally reduces the complexity of the proximal stimulus. Thus, regardless of the orientation of the axis of rotation in the environment, when the axis of rotation is aligned with the symmetry axis, F, through opposite faces of the cube, the orientations of four of the edges of the cube are parallel to the axis of rotation and remain so in the projection throughout rotation. And, when the axis of rotation is aligned with the symmetry axis, $C$, through opposite corners, those two corners remain fixed points in space and in the projection throughout the rotation. The proximal pattern for rotations about these symmetry axes becomes even simpler and, apparently, more memorable when the axis of rotation is also either aligned with or (as in Figure 2) orthogonal to the line of sight. In general, however, the invariance that must be picked up by the perceptual system during a rotation is relatively abstract. The axis of the rotation is not itself visible, and a rotation about a symmetry axis that passes through opposite faces but is oblique in the observer's environment has no visible fixed point. What distinguishes the projections of such a rotation from a rotation about a nonsymmetry axis that is also oblique must be a "higher order" invariance over successive projections in time, such as the invariance in the orientations of the four edges parallel to the axis of rotation.

Second, apart from any specific proximal cues associated with the rotation of a particular object about a particular axis, the perceptual system may directly abstract the axes of greatest symmetry of a three-dimensional object from its two-dimensional projection, much as Gibson $(1966,1979)$ argued that the perceptual system directly picks up the invariants of a motion (in the case of a rotational motion, presumably, the invariant axis of this rotation; see Shepard, 1984). Recent analyses of the kinematic geometry of a rigid body in terms of the Euclidean group for three-dimensional space (Carlton \& Shepard, 1990a) and in terms of the symmetry group of the object (Carlton \& Shepard, 1990b) suggest that when there is an alignment between the axes of a transformation and the axes of the object undergoing the transformation, these axes may become more perceptually accessible.

Our experiment has shown that observers more quickly and accurately compare successive rotations of a particular object-namely, a transparent cube-when the rotations of the object are about axes of symmetry of that object. For a fuller understanding of how the representation of spatial transformations is influenced by both geometrical properties such as symmetry and physical properties such as moments of inertia, studies may be needed that present (a) objects that have different kinds of symmetry, (b) objects that (unlike the cube) have either no axes of symmetry or unequal moments of inertia, and (c) motions about axes that do not pass through the centers of the objects. Symmetry may prove to be only one of a number of properties that affect the perception and representation of object motion.

Further experimentation could also assess the influence of the specific viewing conditions we used, including rotational speed and object transparency. An opaque cube would not be subject to apparent reversals of the direction of rotation. Our subjects either reported that they did not experience such reversals or that their comparison of successive rotations was not hampered by such reversals. Nevertheless, such reversals, which may be more likely to occur for cubes in particular orientations, may have had some subtle effects on performance. Finally, observers in our experiment were required to compare rotations that were presented sequentially. Assessment of memory biases independent of perceptual biases might require experiments in which the motions to be compared are displayed simultaneously or in which the delay between successively presented motions is systematically varied.

In any case, the consistent decreases that we found in speed and accuracy of comparison of rotating cubes as the axes of rotation depart from the canonical axes of the environment or from the symmetry axes of the cube cannot be explained in terms of internalized principles of physics. Rotations about all of the axes we tested were equivalent in terms of physical dynamics; they differed only in their purely geometrical relations to the observer's environmental frame and to the geometrical structure (symmetries) of the cube itself. Hence, our results indicate that purely geometrical constraints influence our perception and representation of the motions of objects in space.

\section{References}

Alluisi, E. A. (1961, December). Lineal inclination in encoding information symbolically on cathode ray tubes and similar displays. ASD Technical Report 61-741, Aeronautical Systems Division, Wright-Patterson Air Force Base, Ohio.

Appelle, S. (1972). Perception and discrimination as a function of stimulus orientation: The oblique effect in man and animals. Psychological Bulletin, 78, 266-278.

Attneave, F., \& Curlee, T. E. (1977). Cartesian organization in the immediate reproduction of spatial patterns. Bulletin of the Psychonomic Society, 10, 469-470.

Attneave, F., \& Olson, R. K. (1967). Discriminability of stimuli varying in physical and retinal orientation. Journal of Experimental Psychology, 74, 149-157.

Bouma, H., \& Andriessen, J. J. (1968). Perceived orientation of isolated line segments. Vision Research, 8, 493-506.

Braine, L. G. (1978). A new slant on orientation perception. American Psychologist, 33, 10-22.

Bryant, P. E. (1969). Perception and memory of the orientation of visually presented lines by children. Nature, 224, 1331-1332. 
Carlton, E. H., \& Shepard, R. N. (1990a). Psychologically simple motions as geodesic paths: I. Asymmetric objects. Journal of Mathematical Psychology, 34, 127-188.

Carlton, E. N., \& Shepard, R. N. (1990b). Psychologically simple motions as geodesic paths: II. Symmetric objects. Journal of Mathematical Psychology, 34, 189-228.

Clark, H. H. (1973). Space, time, semantics and the child. In T. Moore (Ed.), Cognitive development and the acquisition of language. New York: Academic Press.

Cooper, L. A., \& Shepard, R. N. (1973). Chronometric studies of the rotation of mental images. In W. G. Chase (Ed.), Visual information processing (pp. 75-176). New York: Academic Press.

Corballis, M. C., Nagourney, B., Shetzer, L. L., \& Stefanatos, G. (1978). Mental rotation under head tilt: Factors influencing the location of the subjective frame of reference. Perception \& Psychophysics, 24, 263-272.

Corballis, M. C., \& Roldan, C. E. (1975). Detection of symmetry as a function of angular orientation. Journal of Experimental Psychology: Human Perception and Performance, 1, 221-230.

Cutting, J. E. (1986). Perception with an eye for motion. Cambridge, MA: MIT Press/Bradford Books.

Farrell, J. E., \& Shepard, R. N. (1981). Shape, orientation, and apparent rotational motion. Journal of Experimental Psychology: Human Perception and Performance, 7, 477-486.

Franklin, N., \& Tversky, B. (1990). Searching imagined environments. Journal of Experimental Psychology: General, 119, 63-76.

Freyd, J. J. (1983). The mental representation of movement when viewing static stimuli. Perception \& Psychophysics, 33, 575-581.

Freyd, J. J. (1987). Dynamic mental representations. Psychological Review, 94, 427-438.

Friedman, A., Pilon, D. J., \& Gabrys, G. G. (1988, November). Cognitive coordinate systems for mental rotation. Paper presented at the 29th annual meeting of the Psychonomic Society, Chicago.

Gibson, J. J. (1966). The senses considered as perceptual systems. Boston, MA: Houghton Mifflin.

Gibson, J. J. (1979). The ecological approach to visual perception Boston, MA: Houghton Mifflin.

Hinton, G., \& Parsons, L. M. (1981). Frames of reference and mental imagery. In J. Long \& A. Baddeley (Eds.), Attention and performance $I X$ (pp. 261-277). Hillsdale, NJ: Erlbaum.

Hinton, G., \& Parsons, L. M. (1988). Scene-based and viewer-centered representations for comparing shapes. Cognition, 30, 1-35.

Hock, H. S., \& Trombly, C. L. (1978). Mental rotation and perceptual uprightness. Perception \& Psychophysics, 24, 529-533.

Just, M. A., \& Carpenter, P. A. (1985). Cognitive coordinate systems: Accounts of mental rotation and individual differences in spatial abilities. Psychological Review, 92, 137-172.

Kahn, J. I., \& Foster, D. H. (1981). Visual comparison of rotated and reflected random-dot patterns as a function of their positional symmetry and separation in the field. Quarterly Journal of Experimental Psychology, 33A, 155-166.

Kahn, J. I., \& Foster, D. H. (1986). Horizontal-vertical structure in the visual comparison of rigidly transformed patterns. Journal of Experimental Psychology: Human Perception and Performance, 12. 422-433.

Lashley, K. S. (1938). The mechanism of vision: 15 Preliminary studies of the rat's capacity for detailed vision. Journal of General Psychology, 18, 123-193.

Levine, M., Jankovic, I., \& Palij, M. (1982). Principles of spatial problem solving. Journal of Experimental Psychology: General. $111,157-175$.

Leyton, M. (1986a). A theory of information structure: I. General principles. Journal of Mathematical Psychology, 30, 103-160.

Leyton, M. (1986b). A theory of information structure: II. A theory of perceptual organization. Journal of Mathematical Psychology,
$30,257-305$.

Lynch, K. (1960). The image of the city. Cambridge, MA: MIT Press.

Mani, K., \& Johnson-Laird, P. (1982). The mental representation of spatial descriptions. Memory \& Cognition, 10, 181-187.

McBeath, M. K., \& Shepard, R. N. (1989). Apparent motion between shapes differing in location and orientation: A window technique for estimating path curvature. Perception \& Psychophysics, 46, 333-337.

Metzler, J. (1973). Cognitive analogues of the rotation of threedimensional objects. Unpublished doctoral dissertation, Stanford University.

Metzler, J., \& Shepard, R. N. (1974). Transformational studies of the internal representation of three-dimensional objects. In R. L. Solso (Ed.), Theories of cognitive psychology: The Loyola Symposium (pp. 147-201). Potomac, MD: Erlbaum.

Ogilvie, J. C., \& Taylor, M. M. (1958). Effect of orientation on the visibility of fine wires. Journal of the Optical Society of America, $48,628-629$.

Olson, D. R. (1970). Cognitive development: The child's acquisition of diagonality. New York: Academic Press.

Olson, D. R., \& Bialystok, E. (1983). Spatial cognition: The structure and development of mental representations of spatial relations. Hillsdale, NJ: Erlbaum.

Palmer, S. E. (1982). Symmetry, transformation, and the structure of perceptual systems. In J. Beck (Ed.), Organization and representation in perception. Hillsdale, $\mathrm{NJ}$ : Erlbaum.

Palmer, S. E. (1984). The psychology of perceptual organization: A transformational approach. In A. R. Rosenfeld \& J. Beck (Eds.), Human and machine vision. New York: Academic Press.

Palmer, S. E., \& Hemenway, K. (1978). Orientation and symmetry: Effects of multiple, rotational, and near symmetries. Journal of Experimental Psychology: Human Perception and Performance, 4, 691-702.

Parsons, L. M. (1987). Visual discrimination of abstract mirrorreflected three-dimensional objects at many orientations. Perception \& Psychophysics, 42, 49-59.

Post, R. B., \& Chaderjian, M. (1987). Perceived path of oblique motion: Horizontal-vertical and stimulus-orientation effects. Perception, 16, 23-28.

Proffitt, D. R., Gliden, D., Kaiser, M. K., \& Whelan, S. (1988). The effect of configural orientation on perceived trajectory in apparent motion. Perception \& Psychophysics, 435, 465-474.

Rock, I. (1973). Orientation and form. New York: Academic Press.

Rock, I., \& Heimer, W. (1957). The effect of retinal and phenomenal orientation on the perception of form. American Journal of Psychology, 70, 493-511.

Rock, I., \& Leaman, R. (1963). An experimental analysis of visual symmetry. Acta Psychologica, 21, 171-183.

Rudel, R., \& Teuber, H. (1963). Discrimination of direction of line in children. Journal of Comparative and Physiological Psychology, $56,892-898$.

Shepard, R. N. (1981). Psychophysical complementarity. In M. Kubovy \& J. R. Pomerantz (Eds.), Perceptual organization (pp. 279-341). Hillsdale, NJ: Erlbaum.

Shepard, R. N. (1982). Perceptual and analogical bases of cognition. In J. Mehler, E. C. T. Walker, \& M. Garrett (Eds.), Perspectives on mental representation (pp. 49-67). Hillsdale, NJ: Erlbaum.

Shepard, R. N. (1984). Ecological constraints on internal representation: Resonant kinematics of perceiving, imagining, thinking, and dreaming. Psychological Review, 91, 417-447.

Shepard, R. N. (1987). Evolution of a mesh between principles of the mind and regularities of the world. In J. Dupré (Ed.), The latest on the best: Essays on evolution and optimality (pp. 251-275). Cambridge, MA: MIT Press/Bradford Books.

Shepard, R. N. (1988). The role of transformations in spatial cogni- 
tion. In J. Stiles-Davis, M. Kritchevsky, \& U. Bellugi (Eds.), Spatial cognition: Brain bases and development (pp. 81-110). Hillsdale, NJ: Erlbaum.

Shepard, R. N., \& Cooper, L. A. (1982). Mental images and their transformations. Cambridge, MA: MIT Press/Bradford Books.

Shepard, R. N., \& Farrell, J. E. (1985). Representation of the orientation of shapes. Acta Psychologica, 59, 103-121.

Shepard, R. N., \& Hurwitz, S. (1984). Upward direction, mental rotation, and discrimination of left and right turns in maps. Cognition, 18, 161-193.

Shepard, R. N., \& Metzler, J. (1971). Mental rotation of threedimensional objects. Science, 171, 701-703.

Sutherland, N. (1957). Visual discrimination of orientation by octo- pus. British Journal of Psychology, 48, 55-71.

Sutherland, N. (1969). Shape discrimination in rat, octopus, and goldfish: A comparative study. Journal of Comparative and Physiological Psychology, 67, 160-176.

Yin, R. K. (1969). Looking at upside-down faces. Journal of Experimental Psychology, 81, 141-145.

Zimmer, A. (1984). Foundations for the measurement of phenomenal symmetry. Gestalt Theory, 6, 118-157.

Received July 17, 1989

Revision received April 3, 1990

Accepted April 4, 1990

\section{Instructions to Authors}

Authors should prepare manuscripts according to the Publication Manual of the American Psychological Association (3rd ed.). All manuscripts must include an abstract containing a maximum of 960 characters and spaces (which is approximately 100-150 words) typed on a separate sheet of paper. All copy must be double-spaced. Instructions for preparation of abstracts, tables, figures, references, and abbreviations of measurements appear in the Manual. Also, all manuscripts are subject to editing for sexist language. For further information on content, authors should refer to the editorial in the May 1988 issue of the Journal (Vol. 14, No. 2, p. 322). For information on the other three $J E P$ journals, authors should refer to editorials in those journals.

APA policy prohibits an author from submitting the same manuscript for concurrent consideration by two or more journals. APA policy also prohibits duplicate publication, that is, publication of a manuscript that has already been published in whole or in substantial part in another publication. Prior and duplicate publication constitutes unethical behavior, and authors have an obligation to consult journal editors if there is any chance or question that the paper might not be suitable for publication in an APA journal. Authors of manuscripts submitted to APA journals are expected to have available their raw data throughout the editorial review process and for at least 5 years after the date of publication.

Authors will be required to state in writing that they have complied with APA ethical standards in the treatment of their sample, human or animal, or to describe the details of treatment. A copy of the APA Ethical Principles may be obtained from the APA Ethics Office, 1200 17th Street, N.W., Washington, DC 20036.

Anonymous reviews are optional, and authors who wish anonymous reviews must specifically request them when submitting their manuscripts. Each copy of a manuscript to be anonymously reviewed should include a separate title page with authors' names and affiliations, and these should not appear anywhere else on the manuscript. Footnotes identifying the authors should be typed on a separate page. Authors should make every effort to ensure that the manuscript itself contains no clues to their identities.

Five copies of the manuscripts should be submitted. All copies should be clear, readable, and on paper of good quality. A dot matrix or unusual typeface is acceptable only if it is clear and legible. Authors should keep a copy of the manuscript to guard against loss. Mail manuscripts to the Editor, James E. Cutting, Department of Psychology, Uris Hall, Cornell University, Ithaca, New York 148537601.

For the other $J E P$ journals, authors should submit manuscripts (in quadruplicate) to one of the editors at the following addresses: Journal of Experimental Psychology: General, Earl B. Hunt, Department of Psychology N1-25, University of Washington, Seattle, Washington 98195; Journal of Experimental Psychology: Learning, Memory, and Cognition, Keith Rayner, Department of Psychology, Tobin Hall, University of Massachusetts, Amherst, Massachusetts 01003; and Journal of Experimental Psychology: Animal Behavior Processes, Stewart H. Hulse, Editor-Elect, Department of Psychology, Johns Hopkins University, Baltimore, Maryland 21218. When one of the editors believes a manuscript is clearly more appropriate for an alternative journal of the American Psychological Association, the editor may redirect the manuscript with the approval of the author. 